Recepción: 30/10/2018

Aprobación: 06/11/2018

Reseña de libro

DOI: https://doi.org/10.22267/rhec.192222.59

\section{Sepúlveda Delgado, Omaida. EI conocimiento didáctico-matemático del profesor universitario (Tunja: Editorial UPTC, 2018), 368}

\section{Eliécer Aldana Bermúdez}

Universidad del Quindío, Colombia

El libro es resultado de la investigación doctoral de la autora; de modo amplio, en la línea de Historia y prospectiva de la Educación Superior y Formación de Educadores en Iberoamérica, y de manera específica, en la línea de formación de profesores universitarios sobre el Conocimiento Didáctico Matemático (CDM) del profesor, desde el Enfoque Ontosemiótico del Conocimiento y la Instrucción Matemática (EOS), en el campo del Pensamiento Matemático Avanzado (PMA), centrado en particular en el Pensamiento Algebraico, en Álgebra Abstracta, y de forma concreta en Teoría de Grupos.

En este sentido, el objetivo del estudio es caracterizar los conocimientos básicos, que necesitan los estudiantes de formación matemática (Licenciados en Matemáticas, Matemáticos), para realizar la transposición didáctica cuando hacen docencia universitaria en tópicos de Teoría de Grupos, y en particular, los significados que le asignan a este objeto matemático en la búsqueda de su comprensión.

Doctor en Educación Matemática de la Universidad de Salamanca, España. Docente investigador de la Universidad del Quindío, Colombia. Líneas de investigación: Educación matemática, didáctica de la matemática. Correo electrónico: eliecerab@uniquindio.edu.co. Código ORCID: https://orcid.org/0000-0003-1691-2699 
En consecuencia, para lograr este propósito general el marco conceptual pionero en el estudio es el EOS, y para la caracterización de la dimensión epistémica el CDM de los estudiantes de formación matemática sobre el objeto Grupo. En palabras de la autora, en el modelo del CDM (Godino, 2009) esta faceta se encuentra dividida en tres categorías globales del conocimiento sobre el contenido matemático: conocimiento común del contenido y conocimiento ampliado del contenido: estos conocimientos se consideran en la investigación como base para la potenciación o desarrollo de un conocimiento especializado, necesario para la labor de la enseñanza (Pino-Fanetal., 2013a, 2013b).

En relación con el aspecto metodológico, la investigadora presenta un estudio de tipo mixto; porque contempla tanto el enfoque cualitativo como el cuantitativo. La caracterización del significado Global del objeto Grupo, lo desarrolló a partir de un estudio semiótico y documental de tipo histórico, epistemológico y fenomenológico, mediante un estudio en libros de Historia de la Matemática, biografías de autores e investigaciones realizadas en Didáctica del Álgebra, y la técnica del análisis semiótico desarrollada en el EOS. Así mismo, para el diseño e implementación del instrumento para evaluar el CDM de los estudiantes de formación matemática sobre el objeto Grupo, utilizó una metodología orientada a explorar aspectos del CDM desarrollada en el marco del EOS (Godino, 2009; Pino-Fan, Godino y Font, 2013a, 2013b; Vázquez, 2014).

El libro de investigación bajo esta perspectiva epistémica teórica, y metodológica la autora lo presenta, mediante un encuadre epistemológico en cinco capítulos:

En el primer capítulo, centra su atención en el marco general de la investigación en función de una introducción, delimitación del tema, del área problema, formulación del problema, objetivos, justificación en el contexto internacional y nacional, el marco teórico en los aspectos matemáticos, y los elementos conceptuales que van a dar significado al objeto matemático, y la metodología desde las fases, población, variables e instrumentos.

En el segundo capítulo, de manera muy amplia hace un primer análisis de resultados a partir de lo histórico-epistemológico del Álgebra comenzando por su historia y génesis, para entrar en particular en el estudio del objeto Grupo, en función de la estructura matemática que lo configura. 
En el tercer capítulo, realiza un segundo análisis de resultado sobre el diseño de un instrumento para evaluar el CD, plantea una introducción, objetivo del instrumento, construcción, prueba piloto, análisis y versión final del instrumento.

En el cuarto capítulo, desarrolla un tercer resultado en relación con la evaluación del CDM y los instrumentos teóricos y metodológicos que dan sentido a la investigación.

En el quinto capítulo, expone las conclusiones en función de los objetivos de la investigación, sobre la comprensión de los significados del objeto matemático grupo obtenido, de la primera y la segunda fase de investigación, y cómo han sido entendidos estos significados en función del marco conceptual y teórico que caracterizó el estudio, utilizando como lente teórico el CDM y EOS, para mostrar, cómo los profesores universitarios asignan los significados a una estructura matemática cuando hacen una transposición didáctica asertiva. 